\title{
Characteristics of the continuum variability of the nucleus of NGC 4151 in the optical from 1989-1996
}

\author{
N. I. Merkulova, L. P. Metik, and I. I. Pronik
}

\author{
Crimean Astrophysical Observatory, Crimea, Ukraine \\ and Isaac Newton Institute of Chile, Crimean Branch, 98409, p/o Nauchny, Crimea, Ukraine \\ e-mail: nelly@crao.crimea.ua; ipronik@crao.crimea.ua; metik@crao.crimea.ua
}

Received 16 November 2000 / Accepted 24 April 2001

\begin{abstract}
The Structure Function (SF) analysis of variable fluxes of the Seyfert galaxy NGC 4151 obtained during its extraordinary brightening in 1989-1996 in the $U B V R I$ bands is reported. More than 1500 measurements were made in each spectral band. The logarithmic slopes of $S F$ s for all samples of flux observations are found to be different for intranight and extranight flux variations: $0.81-0.96$ and $0.44-0.60$, respectively. The dependence of the $S F$ slopes on the phase of nucleus activity was revealed. The $S F$ slopes increased for intranight variations in accordance with the nucleus brightening and decreased for yearly variations. The processes causing the flux variations evolved during the nucleus brightening in different directions: for intranight variations the process evolved from flicker-noise to extreme shot-noise; for yearly variations - vice versa. The obtained data provide evidence in favor of the supposition that intranight and extranight variations are caused by different sources. As a result, the variable source of the NGC 4151 nucleus during the active phase in the $U B V R I$ bands exhibited characteristics of radio loud AGNs. The highest values of logarithmic slopes of SF for variations on a time scale of years were in the range $0.8 \leq b \leq 1.1$, and for 10-150 day flares they were as high as 2.2 . There was flattening of the optical spectrum with increasing nucleus brightness. The brightness amplitude of the nucleus in the $U$ band reduced to an aperture $D=5^{\prime \prime}$ over 7.3 years increased by a factor of more than 50 . The brightness in the $U$ band varied by a factor of more than 10 per year. The obtained characteristics of the variable source acting in the NGC 4151 nucleus from 1990-1996 do not contradict the model where the increasing nucleus brightening is caused by clouds of synchrotron radiation ejected from the nucleus during its active period.
\end{abstract}

Key words. galaxies: NGC 4151 - Seyfert - active nuclei: optical variability

\section{Introduction}

The continuum variability search of active galactic nuclei (AGNs) is thought to be a useful probe for understanding their nature. Large international monitoring campaigns on some Seyfert galaxies (NGC 3783, NGC 4151, NGC 5548, NGC 7469) have produced a number of fundamental results which provide important constraints on models of the continuum source that cannot be obtained in a single observation. At present the most appropriate model is an accretion disk around a black hole.

The program of simultaneous $U B V R I$ monitoring of nuclei of Seyfert galaxies has been ongoing at the Crimean observatory since 1989. Some results important for the modelling of the AGNs have been obtained. Two types of intranight flares in the variable flux of the NGC 1275 nucleus were revealed by Pronik et al. (1999a, b) using

Send offprint requests to: N. Merkulova,

e-mail: nelly@crao.crimea.ua
1982-1994 observations. Both types of intranight flares were independent of the nucleus brightness averaged by nights. It was supposed that intranight and extranight variations are caused by different sources. Merkulova (2000) showed that two types of intranight flares acted in NGC 4151 and NGC 7469 nuclei, too. The amplitudes of intranight variations were up to $15 \%$.

A new cycle of NGC 4151 nucleus activity beginning from 1990 was observed by Merkulova et al. (1999a,b). They reviewed the variability investigations of the NGC 4151 nucleus published by many authors and studied its extraordinary brightening between February 1990 and June 1996. According to Ulrich et al. (1997), the $U V$ flux $(1440 \AA)$ of the new variable source was increased by a factor 5 in 1993 than it was in 1984-1985. Lyuty \& Doroshenko (1999) noted that a new cycle of brightening of the NGC 4151 nucleus is distinguished from the previous cycle of activity in 1968-1983 in the $U B V$ bands by a higher luminosity by a factor of 2 . Flares of 50-100 days 
duration were observed both at the period of minimum in 1984-1989 and at both cycles of maxima of the nucleus activity. According to Merkulova et al. (1999) the energy distribution, obtained by $U B V R I$ spectral band observations for flux excesses over the 1.5-2.0 years and during the flares, showed a power law form: $F_{\nu} \sim \nu^{\alpha_{\mathrm{pl}}}$. The increase of the power law spectral index $\alpha_{\mathrm{pl}}$ with time from the beginning to the end of the nucleus brightening indicated that there was a flattening of the optical spectrum with time. The data obtained were discussed in the framework of a model where the increase of the nucleus brightening is caused by clouds emitting synchrotron radiation ejected from the nucleus during its active period.

In this paper we present the results of a Structure Function analysis of the variable fluxes. Processes causing the nucleus variability and its evolution with time are considered.

\section{Log of characteristics of observational data}

Observations of the nuclear region of the Seyfert galaxy NGC 4151 have been carried out at the Crimean Astrophysical Observatory with the $1.25 \mathrm{~m}$ telescope using an aperture 20 arcsec in diameter. Over 96 nights, 8 observational runs were made between February 11, 1989 and June 14, 1996. We performed more than 1500 measurements in each of 5 spectral bands of Johnson's $U B V R I$ system simultaneously. One measurement consists of 8 integrations of $10 \mathrm{~s}$ each. Photon statistics corrected for sky background are applied to calculate photometric errors, which were taken to be the same as the rms errors obtained by averaging eight integrations. Taking into account the noise variations of sky background and of a comparison star, we derived the following rms errors for a single observation in the $U B V R I$ bands, respectively: $0{ }^{\mathrm{m}} 011,0 .{ }^{\mathrm{m}} 006,0.007,0{ }^{\mathrm{m}} 007$ and 0.004 . About $50 \%$ of observational nights lasted more than 1 hour; the longest intranight observational run lasted 7.3 hours. The circumstances of the observations were discussed in detail by Merkulova et al. (1999a, b).

The light curves of NGC 4151 in each of the $U B V R I$ bands consist of a slow increase of intensity over almost all the observing time, with 10-150 day outbursts superimposed on it (see Fig. 1). The increase of the nucleus brightness began in February, 1990. The extraordinary long-term brightening continued until June, 1996. The amplitudes of brightening and gradients decreased from the $U$ to the $I$ band. The amplitudes amounted to $2{ }^{\mathrm{m}} 2,1^{\mathrm{m}} 5,0.9,0.8$ and 0.7 in the $U B V R I$ bands, respectively. The variability in the $I$ band was at the level of about $10 \%$ per year. Observations obtained in 199798 with Hubble Space Telescope using a technique of high sensitivity and resolution of near-infrared imaging detected variability of the NGC 4151 nucleus at $1.6 \mu \mathrm{m}$ at the same level: $11 \%$ over 7.3 months (Quillen et al. 2000). The amplitudes of the $U B V R I$ flux variability $F_{\max } / F_{\min }$ for ten day flares were $3.4,1.8,1.4,1.4$ and 1.3 , respectively. The amplitudes of intranight variations were as much as $15 \%$. The amplitudes of all levels of flux variability were significantly higher than the observational errors.

A lot of the observational data permits us to search for the characteristics of the flux of the NGC $4151 \mathrm{nu}$ cleus variability with time, dividing all observational periods into four parts with a duration of 1.5-2.0 years as indicated in Table 1 and in Fig. 1. The duration of each period of time was defined approximately.

The columns of this table show: (1) the number of periods of observation; (2) the starting and ending Julian Dates of the period of observations ( JD $=\mathrm{jd}+$ 2400000 days); (3) the duration of the observations $\mathrm{d} t$, days; (4) the number of observational nights $(n)$; $(5)$ the number of measurements $\left(n_{1}\right)$; $(6)$ the power law spectral slopes of flux excesses for I-IV periods of the nucleus brightening $\left(\alpha_{\mathrm{pl}}\right)$ according to Merkulova et al. (1999a).

Table 1. Characteristics of four observational periods.

\begin{tabular}{llllll}
\hline $\begin{array}{l}\text { Period } \\
\text { of observ. }\end{array}$ & $\begin{array}{l}\text { jd } \\
\text { days }\end{array}$ & $\begin{array}{l}\mathrm{d} t, \\
\text { days }\end{array}$ & $\begin{array}{l}n, \\
\text { nights }\end{array}$ & $\begin{array}{l}n_{1}, \\
\text { points }\end{array}$ & $\alpha_{\mathrm{pl}}$ \\
\hline 1 & 2 & 3 & 4 & 5 & 6 \\
\hline I & $47569-48216$ & 648 & 18 & 190 & -1.17 \\
II & $48216-48764$ & 528 & 22 & 360 & -1.21 \\
III & $48764-49479$ & 522 & 10 & 167 & -0.90 \\
IV & $49479-50249$ & 764 & 46 & 841 & -0.37 \\
\hline
\end{tabular}

\section{Structure function analysis of variable $U B V R I$ fluxes}

\subsection{Properties of structure function and analysis realization}

Press (1978) suggested that the variability in quasars is caused by a process known as "noise". The power spectrum of the simplest of them has a form $g(\nu) \sim \nu^{-\gamma}$. Compact variable AGNs exhibit three types of noise: white-noise $(\gamma=0)$, flicker-noise $(\gamma=1)$, and shot-noise $(\gamma=2)$ (see Terebizh 1993). Such processes are easily revealed by the technique of $\mathrm{SF}$ analysis. In application to AGNs this has been discussed by Hufnagel \& Bregman (1992), Hughes et al. (1992), Lainela \& Valtaoja (1993) and others. The first-order $S F$ is defined as:

$$
S F=<[F(t)-F(t+\mathrm{d} t)]^{2}>
$$

$F(t)$ being the flux at time $t$, and $\mathrm{d} t$ being the time delay (lag) between observations of fluxes $F(t)$ and $F(t+\mathrm{d} t)$, the angular brackets denote an ensemble average. The $S F$ of an "ideal" stationary random process on a logarithmic scale consists of three components: a straight line with a slope $b=\mathrm{d} \log (S F) / \mathrm{d} \log \mathrm{d} t$, which is located between two plateaus. For short time scales, the plateau is just twice the variance of the measurement noise, because it has a zero correlation time scale. The longest correlation time scale $-T_{\max }$ gives the time lag when the $S F$ reaches the 


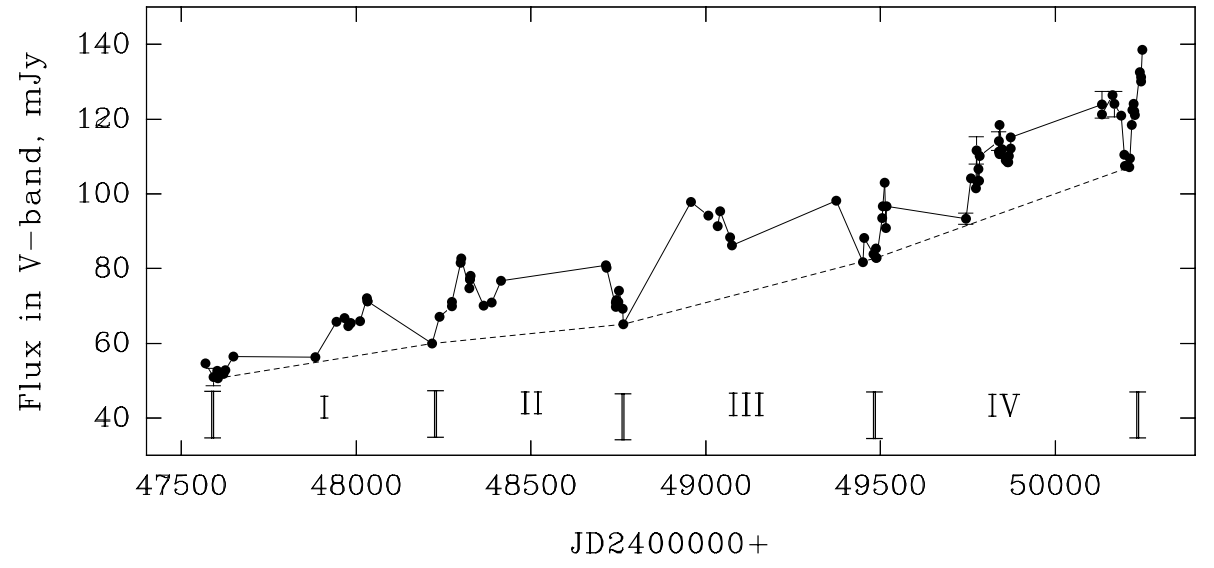

Fig. 1. The light curve of NGC 4151 obtained in the spectral band $V$ using a circular diaphragm $20^{\prime \prime}$ in diameter. The circles are nightly mean values of flux densities in mJy plotted versus the Julian Dates. Bars are rms variations about mean values. Dashed line shows a low boundary of the light curve. Four periods of observations under consideration are indicated by roman numerals. upper plateau with an amplitude equal to twice the variance of the fluctuation. The logarithmic slope " $b$ " characterizes the nature of the process: $b=0$ corresponds to flicker-noise, $b=1$ to shot-noise, $T_{\max }$ characterizes the duration of the flares.

The $S F$ method is related to the autocorrelation function and Fourier power spectrum technique which are more commonly used in the literature. Only methods of $S F$ and a Fourier power spectrum permit one to determine the type of process causing the flux variability. However, the results of analysis using the Fourier power spectrum depend on windowing the sampling whereas the results of analysis by $S F$ do not. The $S F$ realization for the variable flux of the NGC 4151 nucleus was carried out using a special program package by S. G. Sergeev.

Figure 2 shows the $S F$ s of variable fluxes of NGC 4151 in the $U B V R I$ spectral bands, calculated for the whole sample of observations performed from 1989-1996. One can see that $S F$ s do not have a simple "ideal" singleprocess form. The slopes of $S F$ s of intranight variations are essentially higher than those of extranight variations.

The behavior of the $S F$ on time intervals $0.1-1$ days is defined only roughly because of the lack of observations during daylight. We did not consider these intervals in our analysis.

Parameters of the $S F$ s are presented in the columns of Table 2: (1) spectral band; (2), (4) logarithmic slopes " $b_{1}$ " and " $b_{2}$ " of $S F$ for intranight and for yearly variations, respectively; (3), (5) coefficients of correlation " $k_{1}$ " and " $k_{2}$ " between the $\log S F$ and $\log \mathrm{d} t$; regression lines are shown in Fig. 2. The logarithmic slopes " $b$ " of $S F$ on time-scales of hours are equal to $0.81-0.96$ and on timescales of years they are $0.44-0.60$. The confidence levels of the $(\log S F-\log \mathrm{d} t)$ correlations are equal to $1 . T_{\max }$ for intranight process is equal to 1 hour and for yearly variations -4.2 years.

The values of the slopes indicate that the process causing the variations on a time-scale of hours was near to that of shot-noise, but on a time-scale of years it was mixed shot-noise and flicker-noise. This fact permits one to suppose that the sources causing intranight and extranight variations are different. We examined $S F$ s of intranight variations and those on a time--scale of months and years separately.

The high confidence level for the $S F$ parameters was obtained only for the period of 4.2 years $(\log \mathrm{d} t=3.2)$. Confidence levels of the $S F$ parameters for the whole observational period 7.2 years $(\log \mathrm{d} t=3.4)$ were rather low. We suppose that characteristics of weekly and monthly variability over the periods with the lack of observations would be the same as those over our observational periods.

\subsection{Structure function of intranight variability}

Structure function parameters of the intranight variability of the NGC 4151 nucleus are presented in Table 3 separately for I-IV periods of observations. The columns contain: 1) period of observations; 2) spectral band; 3) slope " $b$ "; 4) $T_{\max }$; 5) coefficient of correlation $(k)$ between the values of $\log \mathrm{d} t$ and $\log S F$, obtained for regression lines as shown in Fig. 3. The slopes of $S F$ s are in the range $-0.03 \leq b \leq 1.16$ and $T_{\max } \sim(1.5-5.7)$ hours. The confidence levels of correlations are in the range $0.917-1.000$.

The slopes of the SFs for each period change with wavelength. Table 3 shows that the highest change was in the first period: the slopes decreased from 0.46 for the $U$ band to 0 for the $I$ band. In the second period they are equal to $0.3-0.5$. In the third period all slopes increased to $0.6-0.8$, and in the fourth period they increased to 1.1 . These data show that during the first period the processes causing the flux variation depend on wavelength: mixed shot-noise and flicker-noise for the $U$ band and flicker-noise for the $B V R I$ bands. The dependence of the $S F$ slopes on wavebands was not so clearly expressed during the three following periods. We mentioned the evolution of the process with time in all spectral bands: being near to flicker-noise in the I period, mixed shot-noise and flicker-noise in the II and III periods, and approaching strong shot-noise in the last period.

The obtained results can be interpreted as an indication of increasing intranight nucleus flare activity with increasing nucleus brightness. A clear dependence of the $S F$ slope "b" on waveband is exhibited in the first period but not obvious in the II-IV periods. 
Table 2. Parameters of the $S F \mathrm{~s}$ in the $U B U R I$ bands for all observational samples on different time-scales.

\begin{tabular}{lllll}
\hline \multirow{2}{*}{$\begin{array}{l}\text { spectral } \\
\text { band }\end{array}$} & \multicolumn{2}{c}{ intranight } & \multicolumn{2}{c}{ yearly } \\
\cline { 2 - 5 } & $b_{1} \pm \sigma$ & $k_{1} \pm \sigma$ & $b_{2} \pm \sigma$ & $k_{2} \pm \sigma$ \\
\hline 1 & 2 & 3 & 4 & 5 \\
\hline$U$ & $0.959 \pm 0.081$ & $0.960 \pm 0.021$ & $0.599 \pm 0.057$ & $0.844 \pm 0.043$ \\
$B$ & $0.950 \pm 0.071$ & $0.968 \pm 0.017$ & $0.583 \pm 0.051$ & $0.863 \pm 0.037$ \\
$V$ & $0.930 \pm 0.065$ & $0.972 \pm 0.015$ & $0.526 \pm 0.053$ & $0.827 \pm 0.046$ \\
$R$ & $0.812 \pm 0.080$ & $0.946 \pm 0.027$ & $0.438 \pm 0.054$ & $0.725 \pm 0.069$ \\
$I$ & $0.881 \pm 0.052$ & $0.980 \pm 0.011$ & $0.502 \pm 0.057$ & $0.795 \pm 0.054$ \\
\hline
\end{tabular}

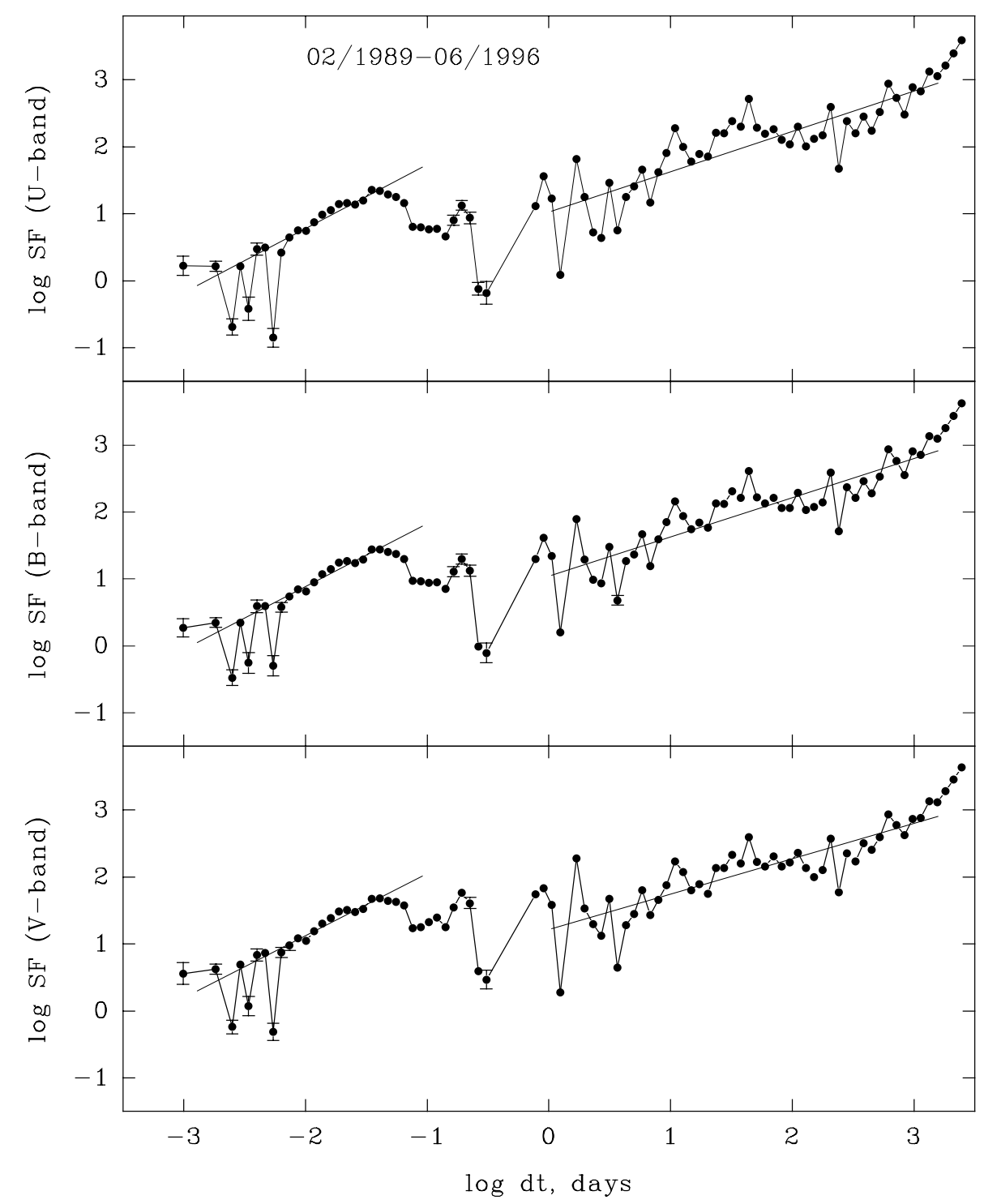

Fig. 2. The Structure Functions for obtained $U B V$ samples. Solid lines are the regression lines (see text). Bars are error bars for each value of the Structure Function, if errors are more than the dimension of signs.

\subsection{Structure Function of extranight flux variability}

Figure 3 shows that $S F$ s for time lags by more than one day curved around $\mathrm{d} t=100$ days

$(\log \mathrm{d} t=2)$, being flatter for yearly variation compared to monthly variation. In the first approximation we neglected this $S F$ curvature and calculated $S F$ parameters for dayly-yearly variations together. Table 4 contains the parameters of the $S F$ s for time lags more than one day for four periods of observation separately: $b$ - logarithmic slope of $S F \mathrm{~s}, k$ - coefficient of correlation between the values $\log S F$ and $\log \mathrm{d} t$, obtained from regression lines (Fig. 3). Table 4 and Fig. 3 show that the $S F_{\text {s }}$ exhibit slopes $0.25 \leq b \leq 1.08$. The confidence level of the correlation equals $0.977-1.000$.

Figure 4 shows the evolution of slopes with time. In the first period the slopes were in the range $0.7-1.1$. In the second period they decline to $0.3-0.7$. In the third period all the slopes increased to $0.6-0.8$ and in the last period they 


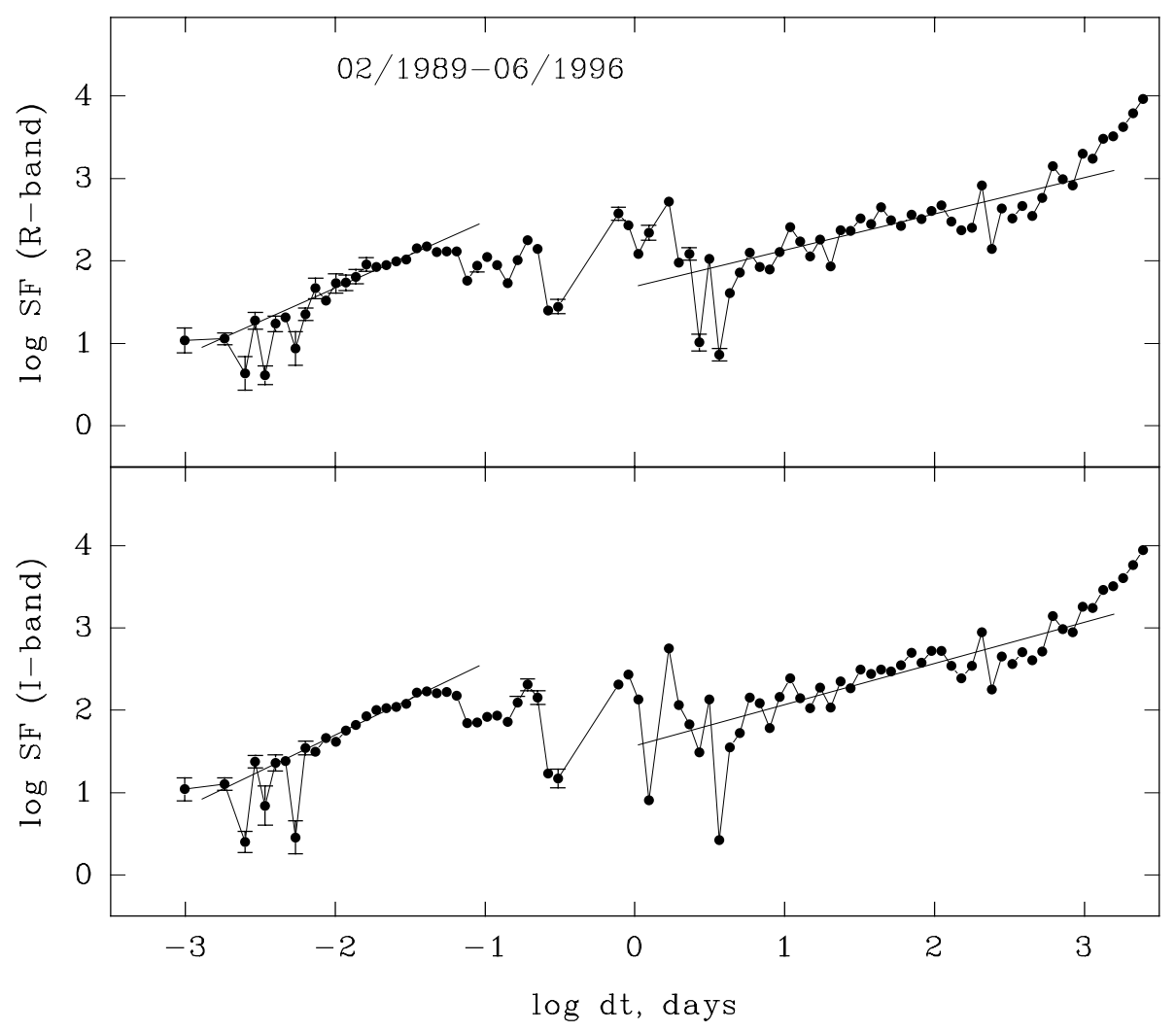

Fig. 2. continued for the $R I$ bands.

Table 3. The Structure Function parameters of intranight variability of the NGC 4151 nucleus for I-IV periods separately.

\begin{tabular}{lllll}
\hline $\begin{array}{l}\text { Period } \\
\text { of observ. }\end{array}$ & $\begin{array}{l}\text { Spectral } \\
\text { region }\end{array}$ & $b$ & $\begin{array}{l}T_{\max }, \\
\text { hours }\end{array}$ & $k$ \\
\hline I & 2 & 3 & 4 & 5 \\
\hline I & $U$ & $0.463 \pm 0.145$ & 2.0 & 0.590 \\
I & $B$ & $0.206 \pm 0.121$ & 2.0 & 0.364 \\
I & $V$ & $0.195 \pm 0.135$ & 2.0 & 0.314 \\
I & $R$ & $0.214 \pm 0.083$ & 2.0 & 0.508 \\
I & $I$ & $-0.03 \pm 0.40:$ & 1.14 & $0:$ \\
\hline II & $U$ & $0.540 \pm 0.064$ & 5.7 & 0.834 \\
II & $B$ & $0.505 \pm 0.063$ & 5.7 & 0.823 \\
II & $V$ & $0.525 \pm 0.063$ & 5.7 & 0.832 \\
II & $R$ & $0.320 \pm 0.099$ & 5.7 & 0.501 \\
II & $I$ & $0.401 \pm 0.069$ & 5.7 & 0.733 \\
\hline III & $U$ & $0.652 \pm 0.039$ & 2.5 & 0.957 \\
III & $B$ & $0.603 \pm 0.027$ & 2.5 & 0.975 \\
III & $V$ & $0.742 \pm 0.039$ & 2.5 & 0.967 \\
III & $R$ & $0.826 \pm 0.052$ & 2.5 & 0.953 \\
III & $I$ & $0.656 \pm 0.077$ & 2.5 & 0.864 \\
\hline IV & $U$ & $1.112 \pm 0.077$ & 1.5 & 0.963 \\
IV & $B$ & $1.112 \pm 0.079$ & 1.5 & 0.962 \\
IV & $V$ & $1.092 \pm 0.081$ & 1.5 & 0.958 \\
IV & $R$ & $1.120 \pm 0.080$ & 1.5 & 0.962 \\
IV & $I$ & $1.162 \pm 0.079$ & 1.5 & 0.965 \\
\hline & & & &
\end{tabular}

decreased to $0.4-0.5$. These data show that the processes causing the general brightness increase from 1989 to 1996 evolved with time. In the first period there was shot-noise in all spectral bands. Then it weakened and approached flicker-noise. In the third period the process was of shotnoise type (coefficient " $b$ " - 0.7-0.8), and at the end of the observational period the process again became mixed shot-noise and flicker-noise $(b \sim 0.4-0.5)$.

Figure 4 shows the dependence of the slopes on spectral bands for each period of time. The extreme values of slope are observed in the $U$ band. This effect is clearest for the first period.

In the second approximation we tried to take into consideration the curvature of the $S F$ around $\log \mathrm{d} t=2$ (see Fig. 3). We calculated $S F$ slopes on a time interval $10 \leq \mathrm{d} t \leq 150$ days $(1.0 \leq \log \mathrm{d} t \leq 2.2)$. Calculated logarithmic slopes $b_{1}$ for corresponding time-scales are presented in Col. 6 of Table 4; the coefficients of correlations $\left(k_{1}\right)$ are in Col. 7 . In all cases the $b_{1}$ were higher than the $S F$ slopes " $b$ " on a time scale of $(1.5-2.0)$ years. One can see that the strongest slopes for flares were observed during the first period of observations when the $b_{1}$ were as much as 2 . We speculate that process of flux variation during the 10-150 day flares was stronger than during the moderate brightening of the nucleus.

\section{Discussion}

\subsection{Summary account}

The analysis of Structure Functions calculated for the $U B V R I$ bands variations of the NGC 4151 nucleus during its extraordinary brightening in 1989-1996 using the 

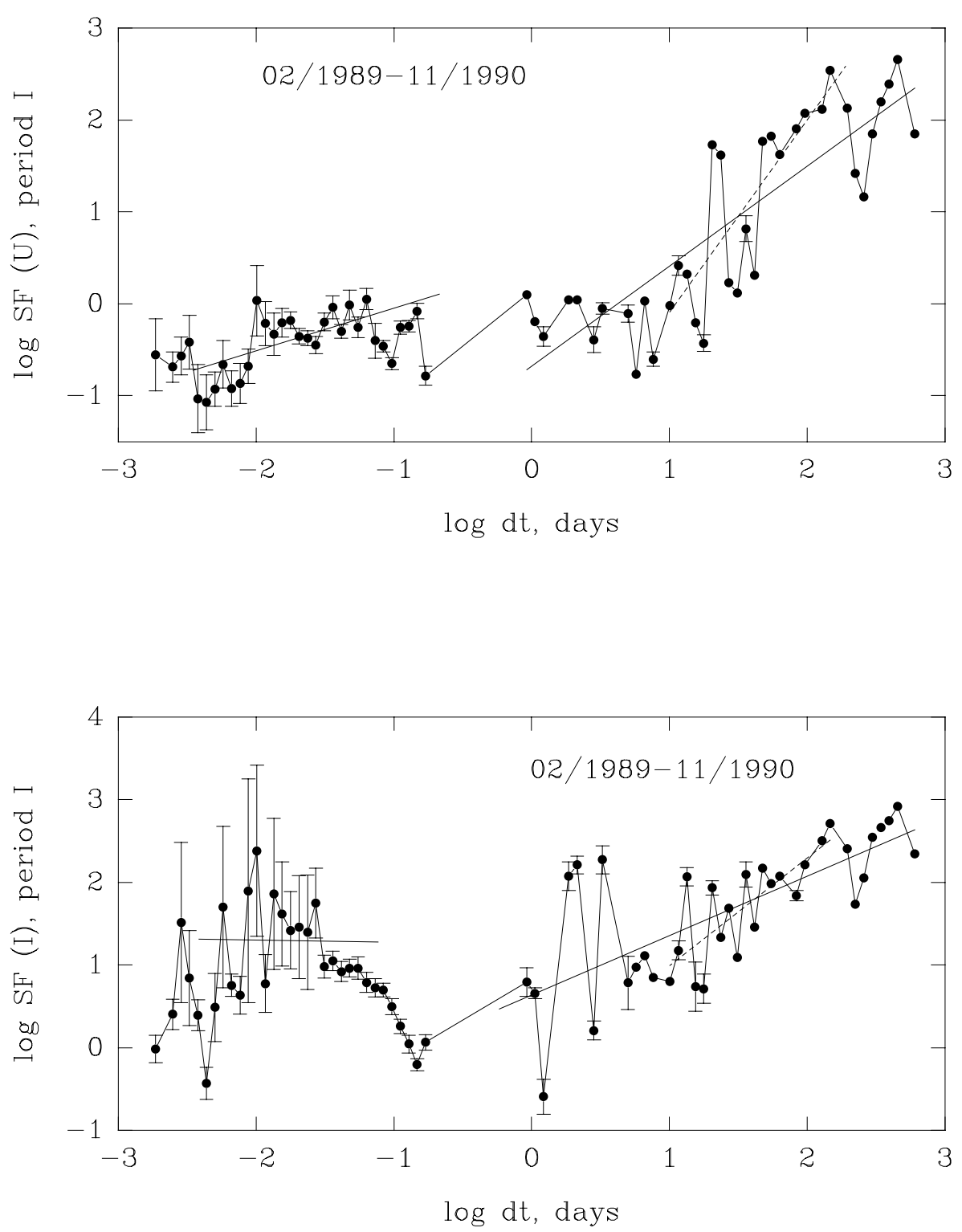

Fig. 3. The Structure Functions for the $U$ and the $I$ bands for different observational periods. Solid lines are regression lines for intranight and extranight flux variations, broken lines are regressions for 10-150 day flares. Error bars for Structure Function values are shown if errors were more than the sign dimension. a) for period I. observational data of 1516 flux measurements in each spectral band led to the following main results:

1. The logarithmic slopes of $S F \mathrm{~s}$ for all periods of observations were different for intranight and extranight variability: $0.81-0.96$ and $0.44-0.60$, respectively. The durations of observed $T_{\max }$ were different, too, being 1 hour and 4.2 years. This indicates that the processes acting on time-scales of hours and years were different: almost pure shot-noise for intranight variations, and mixed shot-noise and flicker-noise for variations on a time-scale of years.

2. The logarithmic slopes of $S F$ s for 10-150 days flares are higher than the $S F$ slopes for the whole period of observations. The difference is highest (factor 2) for the slopes observed during period I.

3 . The evolution of the $S F$ slopes with time was revealed. It developed in different directions for intranight and extranight variations. Intranight processes evolved over 7.3 years from flicker-noise to shot-noise while extranight processes developed in the opposite directions. Flux variations on a time-scale of years became weaker; pulses became less steep when the nucleus brightness increased. At the same time, events on time-scales of less than one day became stronger with time and pulses became steeper and shorter when the nucleus brightening increased. This result shows that intranight and extranight variations are caused by different sources acting in the nucleus.

4. The first period of brightening differs from the three others in the most pronounced dependence of $S F$ slopes on wavelength, both for intranight and for extranight flux variations, being essentially higher for the $U$ band and decreasing to the $I$ band.

\subsection{Discussion}

$S F$ analysis indicates that during the period of 7.3 years brightening (1989-1996) of the NGC 4151 nucleus, different processes acted on different time-scales. There was evolution of the processes and this evolution was in different directions for intranight and extranight variations. 

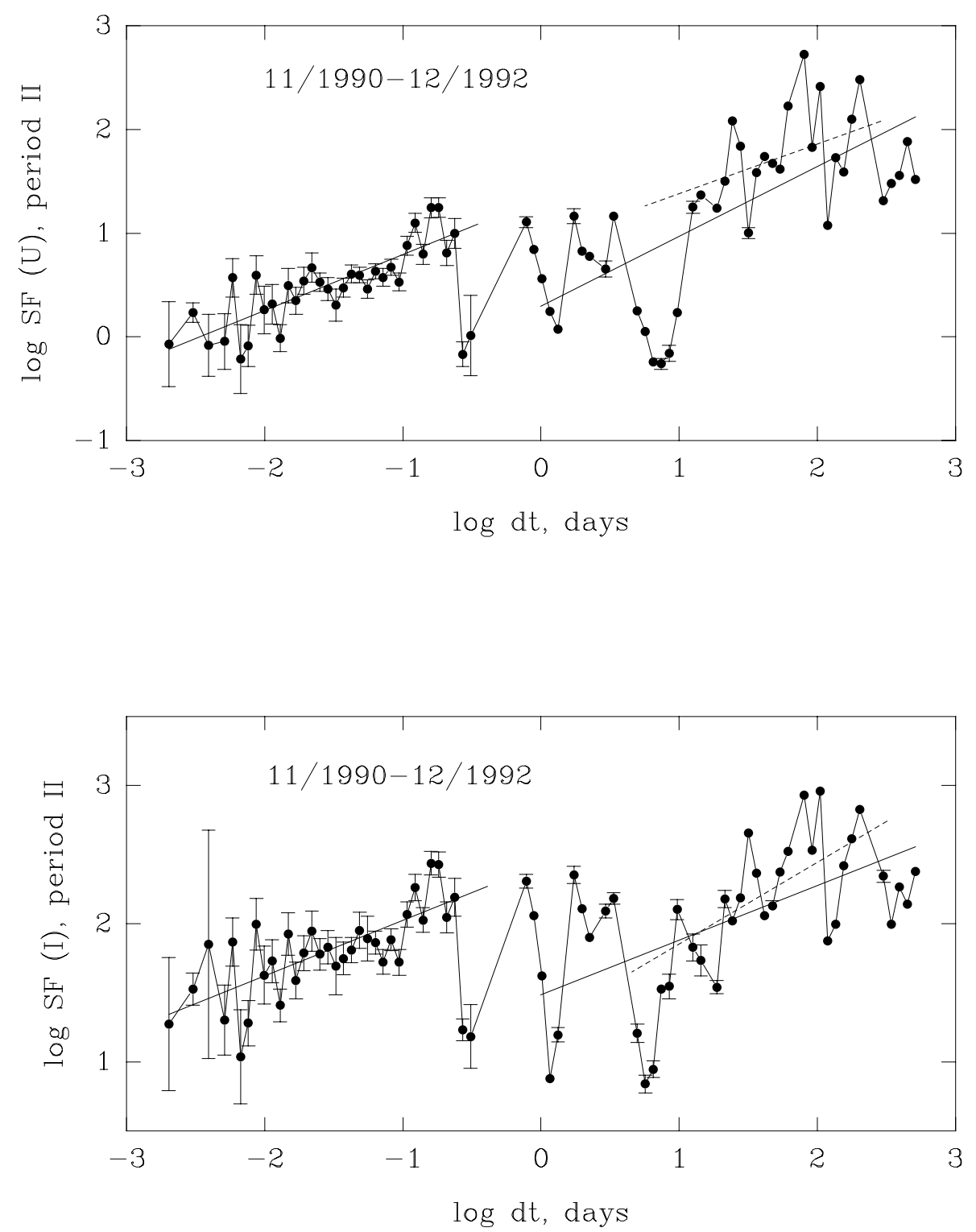

Fig. 3. b) continued for period II.

These data indicate that the sources causing the intranight and extranight variations in the NGC 4151 nucleus are different. We discuss the obtained results for intranight and extranight variations separately.

\subsubsection{Extranight variations}

The logarithmic slopes of the $S F$ (Table 2 and Fig. 2) obtained for extranight $U B V R I$ variations of the NGC 4151 nucleus for the whole sample of 1989-1996 observations are equal to $0.44-0.60$, and are near to those of monthly and yearly optical variations of AGNs obtained by other authors. Hufnagel \& Bregman (1992) analyzed flux density variations in the optical (4400 $\AA$ ) obtained for the five best observed variable AGNs. The nature of the variability is determined using Structure Function analysis. The logarithmic slopes of $S F$ were in the range of $0.3-0.5$. The authors showed that the optical variations are similar for all objects, and are a combination of flicker and shot noise.
Kawaguchi et al. (1998) obtained a $S F$ parameter $b \sim 0.35$ for $2 \leq \log \mathrm{d} t \leq 500$ days for the optical variations of the object $0957+561 \mathrm{~A} / \mathrm{B}$. The calculations led to logarithmic slopes of $S F$ s equal to $0.41-0.49$, which permit them to conclude that the observational slopes of $S F \mathrm{~s}$ of AGNs favored the disk instability model (DI model) .

The nature of weekly, monthly and yearly optical continuum variations of AGNs was discussed by Ulrich et al. (1997) from other point of view. The authors concluded that the continuum flux variability in the optical and UV wavelengths on time-scales from one week to several months observed in low-luminosity AGNs are incompatible with a model wherein this variable component is emitted through viscous effects in an accretion disk. Because the amplitude of the variations at $1400 \AA$ on time-scales of weeks is more than a factor of 2 , at least half of the blue bump luminosity is involved.

The results of the NGC 4151 nucleus observations in the UV favor the point of view of Ulrich et al. (1997). 

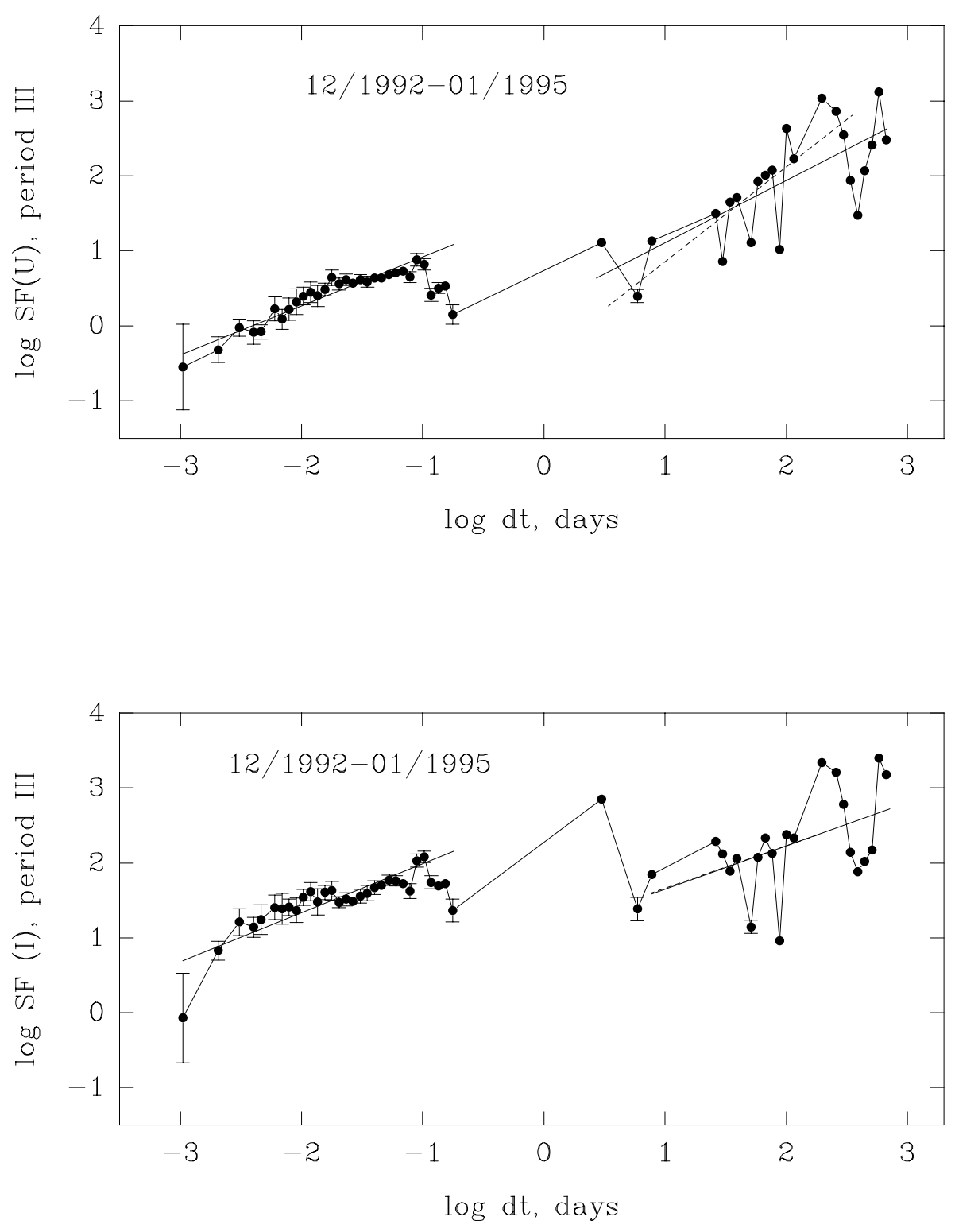

Fig. 3. c) continued for the period III.

A high amplitude of the extrahigh UV variations of the NGC 4151 nucleus was obtained by several authors: Edelson et al. (1996), Kaspi et al. (1996). Paltani \& Walter (1996) analyzed the IUE observations of NGC 4151 complete up to 1991. The UV spectral slope varied considerably, with $\Delta \alpha_{\mathrm{pl}}=1.4$. The authors concluded that neither an accretion disk, nor the optically thin emission model can explain these variations.

The $U B V R I$ light curves of NGC 4151 given by Merkulova et al. (1999a) showed that the greatest amplitude of brightening of the nucleus in 1989-1996 was observed in the $U$ band. The amplitude of flux variability of the NGC 4151 nucleus during the 7.3 years of our observations in an aperture $D=20^{\prime \prime}$ amounts to 2.2 , $1^{\mathrm{m}} 5,0 .{ }^{\mathrm{m}} 9,0^{\mathrm{m}} 8$ and 0.7 in the $U B V R I$ bands, respectively. Reduced to an aperture $D=5^{\prime \prime}$, the amplitude of the $V$ flux varied by a factor more than 9 and the $U$ flux - by a factor more than 50 . The reduction was made using the $U B V$ multiaperture observations of NGC 4151 according to Doroshenko et al. (1998). One may suppose that the yearly brightening was connected with new activity of a variable source of high ultraviolet emission and a high degree of variation. This supposition is supported by our data on the strong shot-noise process revealed in the $U B V$ bands in the first period of the nucleus brightening.

Some alternative models for DI variations in AGNs have been discussed. Daltabuit \& Cox (1972) considered the processes involving the conversion of large-scale kinetic energy to electromagnetic radiation at or near a pair of shock fronts between dense colliding gas clouds.

Shock-in-jet (SJ) models for AGN variability have recently been developed. Marscher (1980) interpreted the radio through optical, and possible X-ray, emission of QSOs and radio weak AGNs in terms of a relativistic jet containing high-energy particles and magnetic field. Marscher \& Gear (1985) presented models including the effects of synchrotron, Compton, and expansion losses as well as variable injection of relativistic electrons and the magnetic 

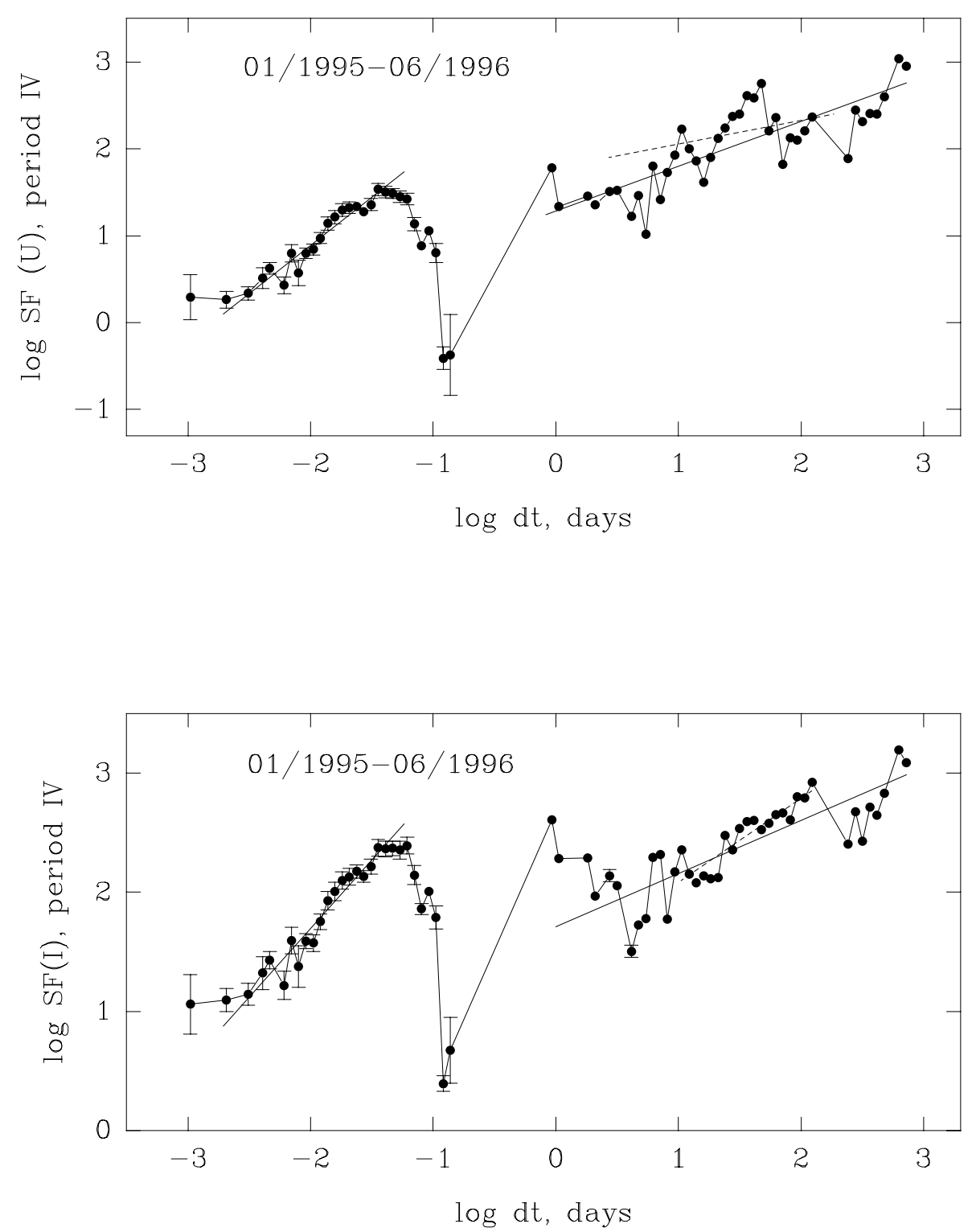

Fig. 3. d) continued for period IV.

field. The observed behavior of AGNs is obtained in a more natural way in a model in which the outburst is due to a shock wave passing through an adiabatic, conical, relativistic jet. The minimum time-scale of variability can be as short as $\sim 1$ day.

Hufnagel \& Bregman (1992) obtained that the slopes of $S F$ for the optical (4400 $\AA$ ) variations of the best observed variable AGNs were inside $0.3-0.5$ exhibiting a mixed shot-noise and flicker-noise process. For the radio region $(4.8-14.5 \mathrm{GHz})$, the flux density variations of these objects revealed a strong shot-noise process $(b \geq 1)$. They explained these results in the framework of models of an inhomogeneous jet of blazars in which shocks propagate along the jet. These models reproduce individual radio outbursts, and may also hold promise to explain optical variations as well. They indicated that the optical and radio emitting regions are physically related, exist on distinct size scales, and excite their synchrotron emitting plasmas differently.
Hufnagel \& Bregman (1992) and other authors pointed out that the flatness of the $S F$ and a decrease of the timescale of variability from radio to optics are characteristic. It is a common opinion that the process causing the optical variability of AGNs is mixed shot-noise and flicker-noise and is weaker than the process causing the radio variability of AGNs. Our SF analysis showed that the process causing the optical fluxes of the NGC 4151 nucleus variability depends on the phase of nucleus activity (see Fig. 4). It was a strong shot-noise type at the beginning of the nucleus brightening (slopes of $S F b \geq 1$, and for flares they were equal $\sim 2$ ). At this time, the process was near to a process being characteristic for radio flux variations of AGNs. But from the beginning to the end of the NGC 4151 nucleus brightening, the process evolved to a mixed shotnoise and flicker-noise. At the end of our observations the process approached flicker-noise, when the nucleus brightness increased: $b \sim 0.4-0.5$. These events have not been 

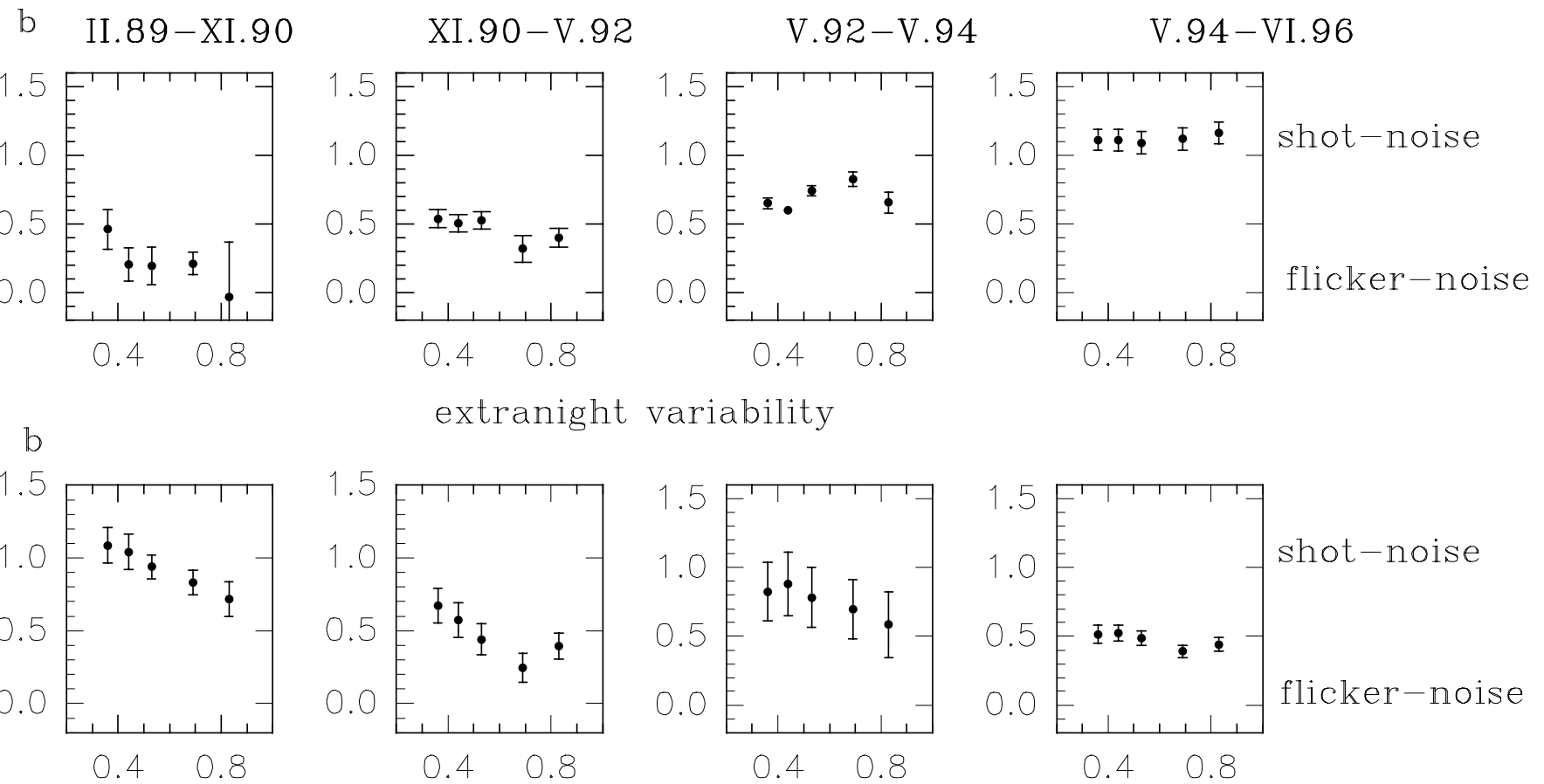

extranight variability
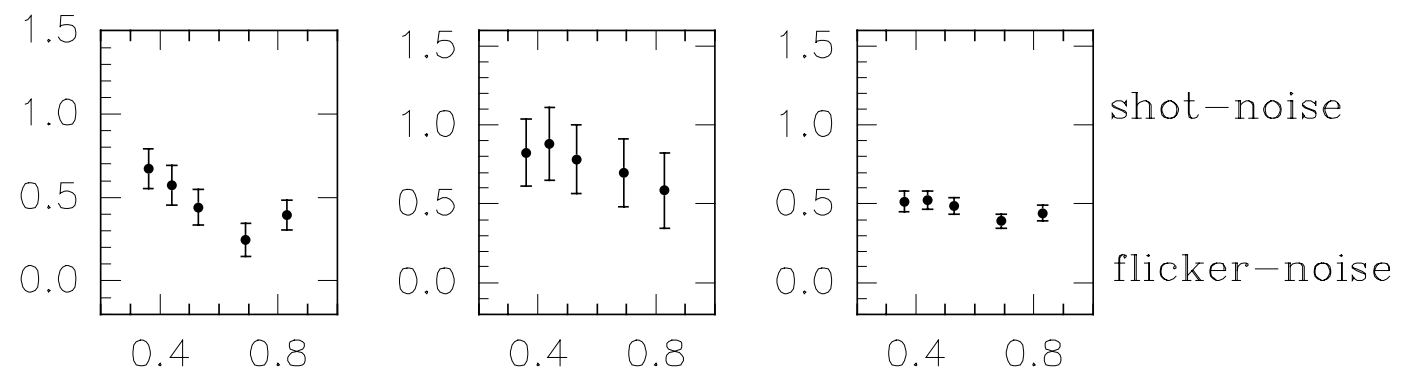

\section{$\lambda(\mathrm{mkm})$}

Fig. 4. Variation of the Structure Function slope " $b$ " with time. Top - for the variability on time-scale of hours, bottom - for yearly variations.

observed for other AGNs because, as a rule, their optical variability was considered for long time periods as a whole, but not for fixed active or nonactive periods. In this case, processes acting at different periods cannot be revealed. Existing phases of a strong shot-noise process for the active period of the NGC 4151 nucleus in the optical region indicated that this process was near to that acting in the radio region of AGNs, and favored the SJ model for its optical flux variability during 1989-1996.

As a rule, SJ models are proposed for blazars and related objects, which show the specific behavior of variations of their spectra. Brown et al. (1989a, b) discussed the continuum variability of blazars from radio to ultraviolet. They obtained that for some of them the correlation between flux level and spectral index, in the sense that the near infrared spectrum flattens when the $J$ band flux density increases. The behavior observed was explained naturally in terms of a single synchrotron component which experienced recurrent bursts of injection, or reacceleration. The steepening of the near-infrared spectrum accompanied by a decrease of flux is attributable to radiative energy losses which affect the higher energy electrons before affecting the lower energy electrons. Quirrenbach et al. (1991) argued that 1-7 days optical variable emission of the object $0716+714$ originates in the jet region. It would be a small jet at a considerable distance from the central engine. Camenzind \& Krockenberger (1992) discussed ra- dio and optical variability in blazars in terms of shocks propagating in collimated relativistic flows.

Hagen-Thorn et al. (1998) analyzed data of the UBVRI 1993-1996 observations of the object OJ 287. They found that the variable sources have the same power-law spectrum in the optical $\left(F_{\nu} \sim \nu^{-1.5}\right)$ irrespective of flux level. In the IR region the spectrum flattens during the flare. Comparison of the outburst of 1994 with those of 1971 and 1983 shows that there is a clear correlation between the power of the outburst and the color indices of the variable component in each event: if the outburst is stronger, the variable source is bluer. At the same time the energy spectrum of relativistic electrons is flatter for a stronger outburst. Hagen-Thorn et al. (1998) suspected a synchrotron self-absorption mechanism for the observed events.

During the brightening of the NGC 4151 nucleus in 1989-1996 the increase of the spectral indices $\alpha_{\mathrm{pl}}$ with time indicated the flattening of the optical spectrum with increasing luminosity of the variable source (Merkulova et al. 1999a). This result is in accordance with the results obtained for blazars by Brown et al. (1989b) and by Hagen-Thorn et al. (1998).

A variable optical source of synchrotron emission in the NGC 4151 nucleus was suspected by Babadzhanjants et al. (1972), Thompson et al. (1979) and Schmidt \& Miller (1980). Observed significant variations in the degree of 
Table 4. Structure Function parameters for yearly $\left(b, T_{\max }\right.$ and $\left.k\right)$ and monthly $\left(b_{1}\right.$ and $\left.k_{1}\right)$ variability of the NGC 4151 nucleus.

\begin{tabular}{lllllll}
\hline $\begin{array}{l}\text { Period } \\
\text { of observ. }\end{array}$ & $\begin{array}{l}\text { Spectral } \\
\text { region }\end{array}$ & $b$ & $\begin{array}{l}T_{\max }, \\
\text { years }\end{array}$ & $k$ & $b_{1}$ & $k_{1}$ \\
\hline 1 & 2 & 3 & 4 & 5 & 6 & 7 \\
\hline I & $U$ & $1.086 \pm 0.122$ & 1.65 & 0.833 & $2.108 \pm 0.404$ & 0.793 \\
I & $B$ & $1.041 \pm 0.122$ & 1.65 & 0.884 & $1.781 \pm 0.442$ & 0.710 \\
I & $V$ & $0.939 \pm 0.084$ & 1.65 & 0.884 & $1.223 \pm 0.340$ & 0.668 \\
I & $R$ & $0.833 \pm 0.084$ & 1.65 & 0.858 & $1.511 \pm 0.259$ & 0.825 \\
I & $I$ & $0.716 \pm 0.119$ & 1.65 & 0.714 & $1.294 \pm 0.275$ & 0.762 \\
\hline II & $U$ & $0.672 \pm 0.117$ & 1.4 & 0.686 & $0.483 \pm 0.310$ & 0.363 \\
II & $B$ & $0.573 \pm 0.121$ & 1.4 & 0.615 & $0.500 \pm 0.275$ & 0.414 \\
II & $V$ & $0.440 \pm 0.106$ & 1.4 & 0.562 & $0.376 \pm 0.258$ & 0.342 \\
II & $R$ & $0.246 \pm 0.101$ & 1.4 & 0.372 & $0.550 \pm 0.253$ & 0.478 \\
II & $I$ & $0.394 \pm 0.088$ & 1.4 & 0.594 & $0.593 \pm 0.247$ & 0.515 \\
\hline III & $U$ & $0.826 \pm 0.215$ & 1.83 & 0.661 & $1.265 \pm 0.398$ & 0.692 \\
III & $B$ & $0.882 \pm 0.233$ & 1.83 & 0.655 & $1.306 \pm 0.450$ & 0.659 \\
III & $V$ & $0.784 \pm 0.219$ & 1.83 & 0.634 & $1.056 \pm 0.400$ & 0.622 \\
III & $R$ & $0.698 \pm 0.215$ & 1.83 & 0.598 & $0.963 \pm 0.318$ & 0.674 \\
III & $I$ & $0.585 \pm 0.240$ & 1.83 & 0.488 & $0.563 \pm 0.464$ & 0.343 \\
\hline IV & $U$ & $0.514 \pm 0.065$ & 1.97 & 0.795 & $0.267 \pm 0.201$ & 0.307 \\
IV & $B$ & $0.524 \pm 0.057$ & 1.97 & 0.838 & $0.366 \pm 0.172$ & 0.458 \\
IV & $V$ & $0.488 \pm 0.054$ & 1.97 & 0.833 & $0.469 \pm 0.144$ & 0.619 \\
IV & $R$ & $0.391 \pm 0.045$ & 1.97 & 0.824 & $0.545 \pm 0.099$ & 0.800 \\
IV & $I$ & $0.443 \pm 0.049$ & 1.97 & 0.838 & $0.704 \pm 0.080$ & 0.907 \\
\hline & & & & & &
\end{tabular}

polarization and its wavelength dependence were among the reasons for this supposition. They obtained that the continuum polarization of the variable source of NGC 4151 varies smoothly throughout the visible, from a value of approximately $1.2 \%$ in the red to approximately $2.5 \%$ at lambda $4000 \mathrm{~A}$ being consistent with the presence of an uniformly polarized synchrotron component. Qualitatively the same wavelength dependence has been observed in other polarized Seyferts. According to Schmidt \& Miller (1980), the observed NGC 4151 continuum can be represented by a combination of a power-law component and a galactic stellar population in proportions approximately $1: 1$ at visual wavelengths.

SJ models include collimated relativistic beaming. As a rule, AGNs of moderate radio emission do not contain such events. However, the data of several observations permit us to suppose that relativistic beaming is possible inside the NGC 4151 central region. Ulvestad et al. (1998) obtained VLBI images of the NGC 4151 central region at wavelengths $6 \mathrm{~cm}$ and $18 \mathrm{~cm}$, achieving a resolution of $\sim 2$ mas and $\sim 5$ mas $(\sim 0.16 \mathrm{pc}$ and $\sim 0.40 \mathrm{pc})$, respectively. They revealed an elongated structure in $\mathrm{PA} \sim 20-75$ degrees, similar to that of the $3 .{ }^{\prime \prime} 5$ radio jet and narrow-line region. The nuclear radio structure at $6 \mathrm{~cm}$ has a length of $\sim 13$ mas (1.0 pc) and a length/width ratio of $\geq 4$ and therefore fulfills one of the classical criteria for a radio jet. However, its radio luminosity is only $\sim 10^{38} \mathrm{ergs} / \mathrm{s}$, several orders of magnitude less than the parsec-scale jets in radio galaxies. Penston et al. (1990), Robinson et al. (1996), Winge et al. (1997) and others showed that the ionization structure of the extended and inner $10 \mathrm{pc}$ narrow line regions of the NGC 4151 nucleus is broadly consistent with photoionization by the AGN radiation field in "an ionization cone" with an opening angle $\sim 120^{\circ}$. They proposed a model of anisotropic emission because relativistic beaming now remains one of the most tenable possibilities.

Pedlar et al. (1993) argued that the UV and milliarcsecond radio structure are collimated along $\mathrm{PA} 50^{\circ}$ on scales $\sim 1 \mathrm{pc}$ and the radio jet is subsequently bent to PA $77^{\circ}$ on a scale of 10 pc. Bicknell et al. (1998) considered radio jets of four Seyfert galaxies, and NGC 4151 is among them. They proposed that "dynamically, Seyfert jets resemble FR1 jets which appear to be initially supersonic and relativistic and to then undergo a transition to turbulent transonic flow. At this transition FR 1 jets are mildly relativistic with $\beta=v / c=0.6-0.7$ ". If this is the case, the polarized variable optical flux of the nucleus which arises in the inner region of relativistic jet can be partly depolarized by turbulent transonic flow.

These data permit us to speculate that extranight variations of the optical continuum flux of the NGC 4151 nucleus may be caused by instabilities in a shock-in-jet event. 


\subsubsection{Intranight variations}

Intranight optical variability is a conventional picture for blazars. There is a strong consensus that activity in a relativistic jet is the best explanation for microvariability and related activity in blazars on time-scales of a few hours (Gonsález-Pérez et al. 1996). Object OJ 287 is one of a few blazars with a very high degree of microvariability which is supposed to be due to the development of a shock and its instabilities (Marscher et al. 1992, Camenzind \& Krockenberger 1992). Wagner et al. (1996), discussing the rapid variability of object S5 $0716+714$ across the electromagnetic spectrum, supposed that the flickering is connected with the plasma processes of particle acceleration.

Analysis of the variability of the NGC 1275 nucleus by Pronik et al. (1999a, b) using observations obtained from 1989-1994 revealed high and low level intranight flares with $S D / F \leq 3 \%$ and $S D / F \geq 3 \%$, where $S D$ is the standard deviation and $F$ is the average flux obtained for each night.

There is now evidence for intranight variation of several Seyfert 1 galaxies, too. Miller \& Noble (1994) observed rapid optical variations of the Seyfert 1 galaxy Akn 120 on a time-scale shorter than an hour. These variations are the most rapid which have been detected for any Seyfert galaxy and clearly demonstrate that radio-quiet AGNs exhibit the phenomenon of microvariability. The authors suggest that these variations are independent of the radio properties of these objects and are thus unlikely to be associated with any disturbance in a relativistic jet. GopalKrishna et al. (1995) supposed that optical microvariability for radio-moderate QSO seems to favor models where flares on accretion discs are responsible for the microvariability.

Intranight optical variations of the NGC $4151 \mathrm{nu}$ cleus were first revealed by Lyuty et al. (1989) using observations at minimum nucleus brightness in 1987 and 1988. They registered $V$ brightness variations of a variable source with $\Delta V \sim 10 \%$ from $15^{\mathrm{m}}-30^{\mathrm{m}}$ and noticed that rapid variations are independent of the level of the nucleus brightness averaged over the night. Merkulova (2000) showed that the two types of intranight flares revealed for the NGC 1275 nucleus by Pronik et al. (1999a, b) also act in the NGC 4151 nucleus.

We observed the evolution of the process causing intranight variability of the nucleus with time during the extraordinary brightening of the nucleus from 1989-1996. At the beginning of the nucleus brightening, the $S F$ slopes on time-scales less than one day were rather low (0.0-0.4) showing that the powering process did not touch the nucleus regions causing the intranight variations. Then evolution of the process was observed from flicker-noise to shot-noise (see Table 3 and Fig. 4), indicating that the powering of the intranight variations was in accordance with the brightening of the nucleus.

Heidt \& Wagner (1996) obtained parameters of SFs for 34 radio selected $\mathrm{BL}$ Lacertae objects in the optical $\mathrm{R}$ band; the slopes of the $S F$ s are within the range
$0 \leq b \leq 2.5$, with a mean value of 0.8 and a dispersion of 0.6 . The typical time-scale lies in the range from 0.5 to 5 days. Our data on microvariability of the optical flux of the NGC 4151 nucleus gave $0 \leq b \leq 1.16$, and do not contradict the results of Heidt \& Wagner (1996), being explained by the standard model where shocks are propagating down a relativistic jet: the SJ model. There will be a high degree of microvariability if the jets are bent and turbulent: the scenario of shocks propagating within the jet entering a turbulent region (Marsher \& Gear 1985; Marscher \& Travis 1991; Wagner 1991). The time-scales observed are close to the lower limit of this model (1 day). From the above mentioned information, there are signs of relativistic beaming in the NGC 4151 central regions (Ulvestad et al. 1998; Penston et al. 1990; Robinson et al. 1996; Winge et al. 1997).

\subsubsection{Extraordinary optical brightening of the NGC 4151 nucleus from 1989-1996 in the framework of the relativistic jet model}

The investigations of the NGC 4151 nucleus variability during its extraordinary brightening between February 1990 and June 1996 by Merkulova et al. (1999a, b) exhibited the characteristics of radio loud AGNs:

1. an energy distribution in the optical flux excesses both in the 1.5-2.0 years variations and in flares with duration 10-150 days showed a power law form: $F_{\nu} \sim \nu^{\alpha_{\mathrm{pl}}}$;

2. an increase in the spectral indices $\alpha_{\mathrm{pl}}$ with time from the beginning to the end of the nucleus brightening indicated a flattening of the optical spectrum;

3. the logarithmic $S F$ slopes of the $U B V R I$ flux variations during the active period were in the range $0.8 \leq$ $b \leq 1.1$, and for 10-150 day flares equal to 2.2 , exhibiting the extreme shot-noise process characteristic for radio variable flux of blazars which were interpreted in the framework of SJ models (Hughes et al. 1992; Lainela \& Valtaoja 1993);

4. high luminosity of ultraviolet emission and a high degree of variation in this emission are revealed. The increase of the nucleus brightening over 7.3 years in the $U$ band reduced to an aperture $D=5^{\prime \prime}$ was by a factor of more than 50 .

The obtained data were discussed in the framework of a model where the increase of the nucleus brightening is caused by the clouds of synchrotron radiation ejected from the nucleus during its active period from 1989-1996.

Figure 5 shows the relation between the slope $\alpha_{\mathrm{pl}}$ of spectral energy distribution in the flux excess during each of four periods of nucleus brightening (from Table 1) and the variable $S F$ parameter " $b$ " for extranight $U$ variations (from Table 4). One can see an inverse correlation of these values. The increase of the spectral index $\alpha_{\mathrm{pl}}$ was proposed as resulting from increase of the optical depth of the clouds of relativistic electrons, or the accumulation of higher energy electrons. The decrease of the $S F$ parameter " $b$ " can be caused by the same: increasing optical depth 


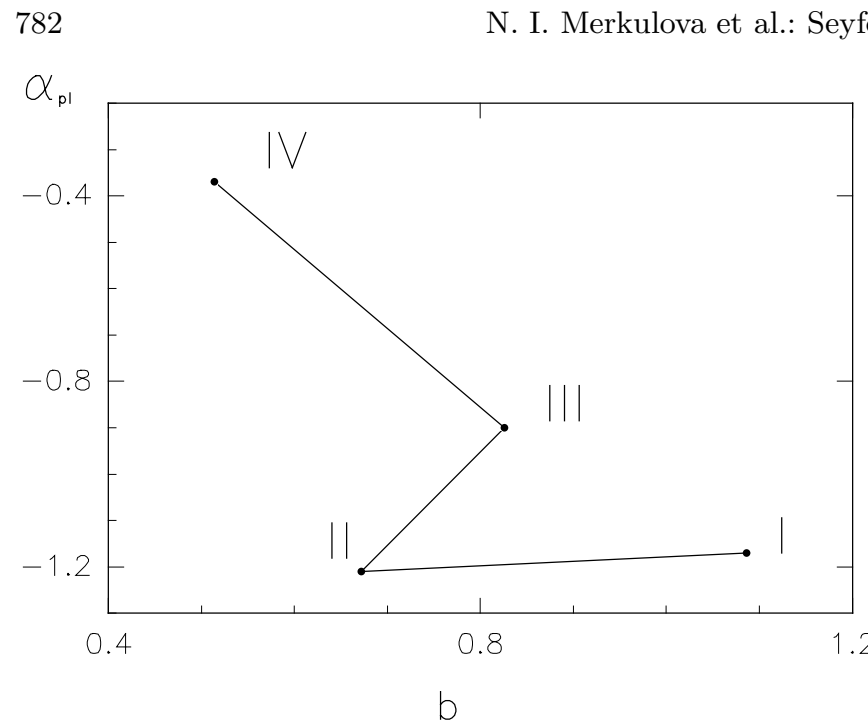

Fig. 5. Inverse correlation of the spectral slopes $\alpha_{\mathrm{pl}}$ with the $S F$ slopes " $b$ ".

of the emission region, or the accumulation of electrons of higher energy leading to smoothing of pulses of light variability.

\section{Conclusions}

Using the Structure Function analysis of the $U B V R I$ variability of the nucleus of the Seyfert galaxy NGC 4151 during its extraordinary brightening from 1989-1996 the following results were obtained:

1. We argued that the processes of intranight and extranight flux variations are caused by different sources. The processes in both cases evolved from the beginning to the end of the brightening but in different directions: for intranight variations the process evolved from flickernoise to shot-noise, for yearly variations - vice versa. The evolution of the intranight process from flicker-noise to shot-noise was in accordance with the increase of the nucleus brightness. At the same time, the increase of nucleus brightness was accompanied by the evolution of extranight process from shot-noise to flicker-noise.

2. The source causing the nucleus NGC 4151 brightening was a source of high ultraviolet emission and of a high degree of variations. The increase of the nucleus brightening in an aperture $D=5^{\prime \prime}$ over 7.3 years in the $V$ band was by a factor of 9 and in the $U$ band it was by factor of more than 50 .

3. The current opinion, that $S F$ slopes of optical flux variations on time-scales of months and years are about $0.3-0.5$ and that they are always less than the $S F$ slopes for radio flux variations in AGNs, is not correct. We found that the slopes depend on the phase of nucleus activity. Our data showed that during the active phase the slopes were equal to 1.0 and during the flares they increased to 2.0 .
4. The slopes of the spectral energy distribution in the $U B V R I$ region $\left(\alpha_{\mathrm{pl}}\right)$ are found to be in anticorrelation with the $S F$ slopes " $b$ " of variable flux. One possible explanation of this fact is that the increase of the nucleus brightness is caused by clouds emitting synchrotron radiation ejected from the nucleus during its active period.

Acknowledgements. We thank Dr. V. I. Pronik for helpful discussion and Dr. S. G. Sergeev for the $S F$ program package.

The research described in this publication was made possible in part by Award No. UP1-2116 of the U.S. Civilian Research \& Development Foundation for the Independent States of the Former Soviet Union (CRDF).

\section{References}

Babadzhanjants, M. K., Hagen-Thorn, V. A., \& Ljutiy, V. M. 1972, Astrofizika, 8, 509

Bicknell, G. V., Dopita, M. A., Tsvetanov, Z. I., et al. 1998, ApJ, 495, 680

Brown, L. M. J., Robson, E. I., Gear, W. K., et al. 1989a, ApJ, 340, 129

Brown, L. M. J., Robson, E. I., Gear, W. K., \& Smith, M. G. 1989b, ApJ, 340, 150

Camenzind, M., \& Krockenberger, M. 1992, A\&A, 255, 59

Daltabuit, E., \& Cox, D. 1972, ApJ, 173, L13

Doroshenko, V. T., Lyutyi, V. M., \& Shenavrin, V. I. 1998, Pis'ma v Rus. Astron. J., 24, 197

Edelson, R. A., Alexander, T., Crenshaw, D. M., et al. 1996, ApJ, 470, 364

González-Peréz, J. N., Kidger, M. R., \& de Diego, J. A. 1996, A\&A, 311, 57

Gopal-Krishna, Ram Sagar, \& Pane J. Witta 1995, MNRAS, 274,701

Hagen-Thorn, V. A., Marchenko, S. G., Takalo, L. O., et al. 1998, A\&AS, 133, 353

Heidt, J., \& Wagner, S. J. 1996, ApJ, 305, 42

Hufnagel, B. R., \& Bregman, J. N. 1992, ApJ, 386, 473

Hughes, P. A., Aller, H. D., \& Aller, M. F. 1992, ApJ, 396, 469

Kaspi, S., Maoz, D., Netzer, H., et al. 1996, ApJ, 470, 336

Kawaguchi, T., Mineshige, S., Umemura, M., \& Turner, E. L. 1998, ApJ, 504, 671

Lainela, M., \& Valtaoja, E. 1993, ApJ, 416, 485

Lyuty, V. M., \& Doroshenko, V. T. 1999, Pis'ma v Rus. Astron. J., 25, 403

Lyuty, V. M., Aslanov, A. A., Volkov, L. M., et al. 1989, Pis'ma v Rus. Astron. J., 15, 579

Marscher 1980, ApJ, 235, 386

Marscher, A. P., \& Gear, W. K. 1985, ApJ, 298, 114

Marscher, A. P., \& Travis, J. P. 1991, in Variability of Active Galactic Nuclei, ed. R. H. Miller, \& P. Wiita (Cambridge Univ. Press), 153

Marscher, A. P., Gear, W. K., \& Traves, J. P. 1992, in Blazar Variability, ed. M. Valtonen, \& E. Valtaoja (Cambridge University Press), 85

Merkulova, N. I. 2000, AJ, 119, 631

Merkulova, N. I., Metik, L. P., \& Pronik, I. I. 1999a, AJ, 117, 2177

Merkulova, N. I., Metik, L. P., \& Pronik, I. I. 1999b, in Activity in galaxies and related phenomena, Proc. IAU Symp., 194, 436 
Miller, H. R., \& Noble, J. C. 1994, International Astrophysics Conference on Mass-Transfer Induced Activity in Galaxies, 59

Paltani, S., \& Walter, R. 1996, A\&A, 312, 55

Pedlar, A., Kukula, M. J., Longley, D. P., et al. 1993, MNRAS, 263,471

Penston, M. V., Robinson, A., Alloin, D., et al. 1990, A\&A, 236,53

Press, W. H. 1978, Comments on Astrophys., 7, 103

Pronik, I. I., Merkulova, N. I., \& Metik, L. P. 1999a, AJ, 117, 2141

Pronik, I. I., Merkulova, N. I., \& Metik, L. P. 1999b, A\&A, 351,21

Quillen, A. C., Shanna Shaked, Alonso-Herrero, A., et al. 2000, ApJ, 532, L17

Quirrenbach, A., Witzel, A., Wagner, S., et al. 1991, ApJ, 372, L71
Robinson, A., Axon, D. J., \& Vila-Vilaro, B. 1996, Vistas in Astronomy, 40, part 1, 77

Schmidt, G., \& Miller, J. 1980, ApJ, 240, 759

Terebizh, V. Yu. 1993, Time Series Analysis in Astrophysics ("Nauka" Publication, Moskva), 389

Thompson I., \& Landstreet, J., et al. 1979, ApJ, 229, 909

Ulvestad, J. S., Roy, A. L., Colbert, E. J. M., \& Wilson, A. S. 1998, ApJ, 496, 196

Ulrich, M.-H., Maraschi, L., \& Urry, C. M. 1997, ARA\&A, 35, 445

Wagner, S. J. 1991, in Variability of Active galaxies, ed. W. J. Dusche, S. J. Wagner, \& M. Camenzind, Proc. Heidelberg (Germany, Springer-Verlag), 163

Wagner, S. J., Witzel, A., Heit, J., et al. 1996, AJ, 111, 2187

Winge, C., Axon, D. J., Macchetto, F. D., \& Capetti, A. 1997, ApJL, 487, 121 